\title{
Ionized outflows in local luminous AGN: Density and outflow rate
}

\author{
R. Davies ${ }^{1}\left(\mathbb{D}\right.$, D. Baron ${ }^{2}$, T. Shimizu ${ }^{1}$ and H. Netzer ${ }^{2}$ \\ ${ }^{1}$ Max-Planck-Institut für extraterrestrische Physik, Postfach 1312, 85741, Garching, Germany \\ ${ }^{2}$ School of Physics and Astronomy, Tel-Aviv University, Tel Aviv 69978, Israel
}

\begin{abstract}
We use the LLAMA survey to study the density and outflow rate of ionized gas in a complete volume limited sample of local $(<40 \mathrm{Mpc})$ luminous $\left(43.0<\log L_{\mathrm{AGN}}(\mathrm{erg} / \mathrm{s})<44.5\right)$ AGN selected by very hard 14-195 keV X-rays. The detailed data available for this survey enable us to measure the density of the outflowing ionized gas in the central $300 \mathrm{pc}$ of these AGN using three different and independent methods (the standard [SII] doublet ratio; a method comparing [OII] and [SII] ratios that include auroral and transauroral lines; and a recently proposed method based on the ionization parameter). For each method there is, as expected, a modest spread of densities among the AGN in the sample. But remarkably, the median densities for each method differ hugely, by an order of magnitude from below $400 \mathrm{~cm}^{-3}$ to almost $5000 \mathrm{~cm}^{-3}$. We discuss how the derived densities can be reconciled, and what the impact is on the implied outflow rate.
\end{abstract}

Keywords. Galaxies: active, Galaxies: ISM, Galaxies: nuclei, Galaxies: Seyfert

\section{Introduction}

That outflows driven by star formation and AGN play a fundamental role in the evolution of galaxies is undisputed. This is highlighted by cosmological models of galaxay evolution, which demonstrate the role that outflows are expected to have in order for such models to reproduce observed galaxy scaling relations (Somerville \& Davé 2015). For these models to halt accretion onto the central supermassive black hole and quench star formation, the kinetic power of the gas outflow should be typically $5 \%$ of the AGN bolometric luminosity (Springel et al. 2005). Whether this is achieved through ionized outflows or cool outflows of neutral and molecular gas is not yet established (Veilleux et al. 2020). As pointed out by Harrison et al. (2018), a large part of this uncertainty is linked to the density of the gas in ionized outflows. The reason is simply that the mass $M_{\text {out }}$ of ionized gas derived from a measurement of line luminosity $L_{\text {line }}$ is inversely proportional to the density $n_{\mathrm{e}}$ adopted: $M_{\text {out }} \propto L_{\text {line }} /\left(\gamma_{\text {line }} n_{\mathrm{e}}\right)$, where $\gamma_{\text {line }}$ is the volume emissivity. In the literature, a very wide range of densities has been assumed, adopted, or measured. Often, the density is estimated using the [SII] doublet ratio. This is applicable up to $\sim 10^{4} \mathrm{~cm}^{-3}$ for stellar HII regions; but may be biased in AGN photoionized gas because the high energy photons penetrate deep into the clouds and create a partially ionized zone, which is responsible for the enhanced emisson from low excitation lines around AGN. The impact of this on the density one derives has recently been assessed by Davies et al. (2020).

The LLAMA (Local Luminous AGN with Matched Analogues) survey (Davies et al. 2015) survey of active and inactive galaxies is an ideal sample in which to assess the true density of gas in ionized outflows because it enables the measurement to be made in several different ways for the same galaxies. The sample was taken from the all-sky flux

(C) The Author(s), 2021. Published by Cambridge University Press on behalf of International Astronomical Union. This is an Open Access article, distributed under the terms of the Creative Commons Attribution licence (http://creativecommons.org/licenses/by/4.0/), which permits unrestricted re-use, distribution, and reproduction in any medium, provided the original work is properly cited. 

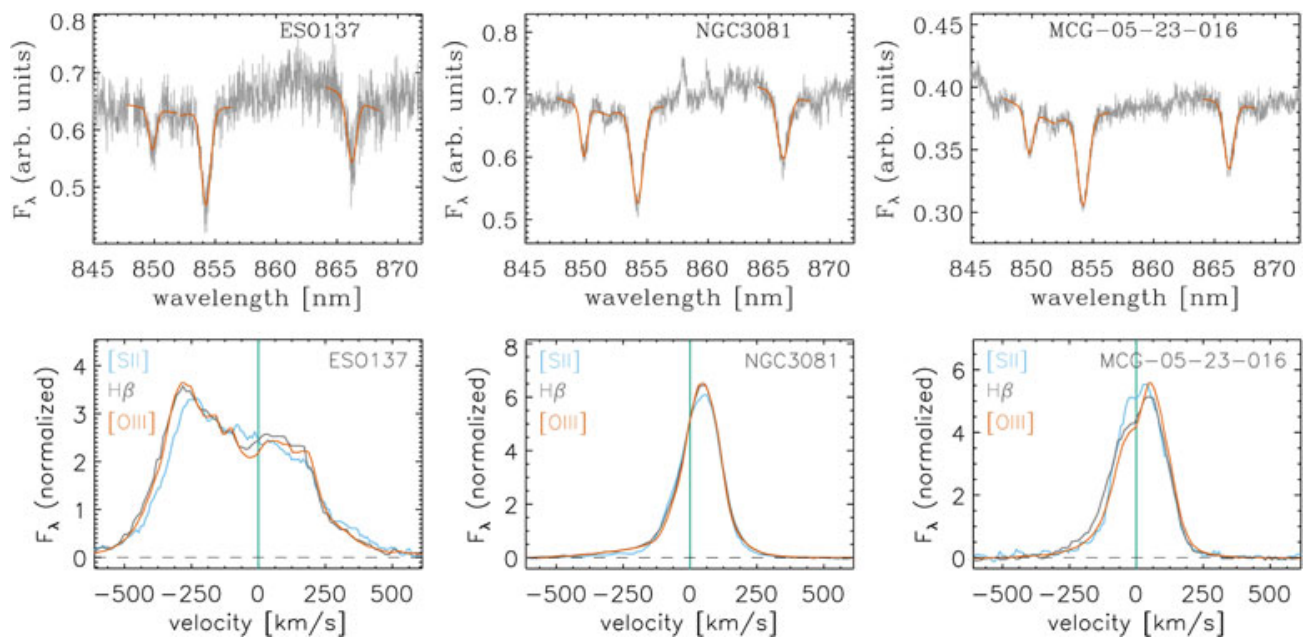

Figure 1. Examples of the CaII triplet stellar absorption (top row) used as the velocity reference for the more complex emission line profiles (bottom row). These are ordered in terms of luminosity, from $L_{\mathrm{AGN}}=10^{43.4} \mathrm{erg} \mathrm{s}^{-1}$ for ESO 137-G034 (left), to $10^{44.1} \mathrm{erg} \mathrm{s}^{-1}$ for NGC 3081, and $10^{44.3} \mathrm{erg} \mathrm{s}^{-1}$ for MCG-05-23-016 (right).

limited 14-195 keV 58-month Swift BAT survey in such a way as to create a volume limited sample of active galaxies that is as unbiased as possible, for detailed study using optical spectroscopy and adaptive optics integral field near-infrared spectroscopy. The sole selection criteria were $z<0.01$ (corresponding to a distance of $\sim 40 \mathrm{Mpc}$ ), $\log L_{14-195 \mathrm{keV}}$ $\left[\mathrm{erg} \mathrm{s}^{-1}\right]>42.5$ (using redshift distance), and $\delta<15^{\circ}$ so that they are observable from the VLT. This yielded 20 AGN. A set of inactive galaxies were selected to match them in terms of host galaxy type, mass (using H-band luminosity as a proxy), inclination, presence of a bar, and distance. Although small, this volume limited sample is sufficient for detailed studies of emission line ratios, the molecular and ionized gas kinematics and distributions, as well as the stellar kinematics and populations, in the nuclear and circumnuclear regions. And the ability to compare the results to a matched sample of inactive galaxies has been essential in many of the studies so far, including the analysis presented here. Here we focus on the optical spectroscopy from Xshooter (Vernet et al. 2011) of a subset comprising Seyfert 2 and inactive galaxies, using spectra extracted from a $1.8^{\prime \prime} \times 1.8^{\prime \prime}$ aperture that corresponds to sizes of $200-350 \mathrm{pc}$ at the distances of the targets.

\section{Emission line characteristics}

This section focusses on whether the AGN emission line profiles are dominated by the interstellar medium or by the outflow. An important ingredient to this is an accurate velocity reference for the emission lines, for which we make use of the Ca II triplet lines around $860 \mathrm{~nm}$. As can be seen from the examples in Fig. 1, these stellar absorption features provide a robust measurement of the systemic velocity because they are strong, well defined, and unbiased by interstellar absorption. The emission lines are rather complex, exhibiting a range of characteristics illustrated by the examples given in the figure. The [SII], $\mathrm{H} \beta$, and [OIII] lines shown have remarkably similar profiles, which do not typically peak at the systemic velocity. In ESO 137-G034, the blue side of the profile is stronger, peaking more than $250 \mathrm{~km} \mathrm{~s}^{-1}$ from systemic, and there is a suggestion of a dip around the systemic velocity. In NGC 3081, without a velocity reference one might conclude that the line peaks at systemic and has a blue wing; but the peak is in fact offset to the 
red side by almost $50 \mathrm{~km} \mathrm{~s}^{-1}$. In MCG-05-23-016, the line profile is centered around the systemic velocity but shows a dip or break at that velocity. These features can all be naturally reproduced by models of biconical outflow.

The emission line profiles for the inactive galaxies are qualitatively different: the peak is always close to systemic (the median offset is only $11 \mathrm{~km} \mathrm{~s}^{-1}$ ), although it is often accompanied by a weak wing. In contrast, for the active galaxies it is the 'wing' that dominates the profile. Our conclusion here is that, in contrast to some observations where a 'core+wing' decomposition may make sense, this is neither possible nor meaningful here. These nuclear measurements of local luminous AGN are fully dominated by AGN photoionized outflow, and any systemic component related to the host galaxy disk is negligible. So, when measuring the density of the outflowing gas, we should integrate over the whole line profile.

\section{Comparison of measured densities}

In this section we compare the densities $\left(n_{\mathrm{e}} \sim n_{\mathrm{p}} \sim n_{\mathrm{H}}\right.$ in fully ionized gas) we find for the ionized gas measured in three rather different ways. Additional details about the methods, and of the line fitting and modelling can be found in Davies et al. (2020).

The most commonly used tracer of $n_{\mathrm{e}}$ uses the [SII] $\lambda 6716,6731 \AA$ doublet, which only requires a measurement of the ratio of two strong emission lines in a convenient and clean part of the optical spectrum. The physics of the excitation and de-excitation means that density - covering a range commonly found in H II regions - dominates the emitted line ratio; and because the lines are necessarily close in wavelength, the result is unaffected by extinction. These lines are well fitted in our data, yielding a median value of $n_{\mathrm{e}} \sim 350 \mathrm{~cm}^{-3}$ for the AGN and $190 \mathrm{~cm}^{-3}$ for the inactive galaxies.

An alternative method was proposed by Holt et al. (2011) as a way to avoid the limitations of the [SII] doublet ratio. In particular it is sensitive to high densities because it uses the transauroral lines [SII] $\lambda 4069,4076$ and the auroral lines [OII] $\lambda 7320,7331$ which have higher critical densities. These are used together with the stronger lines to give the ratios $[\mathrm{SII}] \lambda(4069+4076) /[\mathrm{SII}] \lambda(6716+6731)$ and $[\mathrm{OII}] \lambda(3726+3729) /[\mathrm{OII}] \lambda(7320+7331)$. How these lines are used differs fundamentally from the standard doublet ratio above: advantageously, the lines within each doublet are summed, and the ratios of the total flux in the doublets are compared to photoionization models. Such models take into account internally they way in which $n_{\mathrm{e}}$ (on which the emitted lines depend) varies through the cloud, and hence how the resulting cumulative line ratios are related to $n_{\mathrm{H}}$. In this way the method traces $n_{\mathrm{H}}$, but typically one equates that with $n_{\mathrm{e}}$ as for fully ionized gas. This means the method is dependent on the photoionization model; and also, because of the wide wavelength range covered by the lines, on extinction and the choice of extinction model. It is notable that a grid of models for $n_{\mathrm{e}}$ and $A_{\mathrm{V}}$ has almost orthogonal axes, and comparable dependency on both line ratios. The main limitation of this method is the weakness of the auroral and transauroral lines, which means it can only be applied to high signal-to-noise data. Using this method, the the median value for our data is $n_{\mathrm{e}} \sim 1900 \mathrm{~cm}^{-3}$.

A new method developed by Baron \& Netzer (2019) is based on the definition of the ionization parameter as the number of ionising photons per atom. Re-arranging the definition gives $n_{\mathrm{H}} \propto L_{\mathrm{AGN}} r^{-2} U^{-1}$, so that the density can be derived from the AGN luminosity, the distance of the ionized gas from the AGN, and the ionization parameter. At the same time, Baron \& Netzer (2019) showed that, observationally, $U$ can be derived from the strong line ratios $\mathrm{N}[\mathrm{II}] / \mathrm{H} \alpha$ and $[\mathrm{OIII}] / \mathrm{H} \beta$. Alternatively, for luminous AGN and/or clouds that are close to the AGN, one can adopt a limiting value of $U=0.01$ (Dopita et al. 2002). This method is widely applicable, and well suited to spatially resolved data. However, one needs to be aware of the impact of uncertainties in 


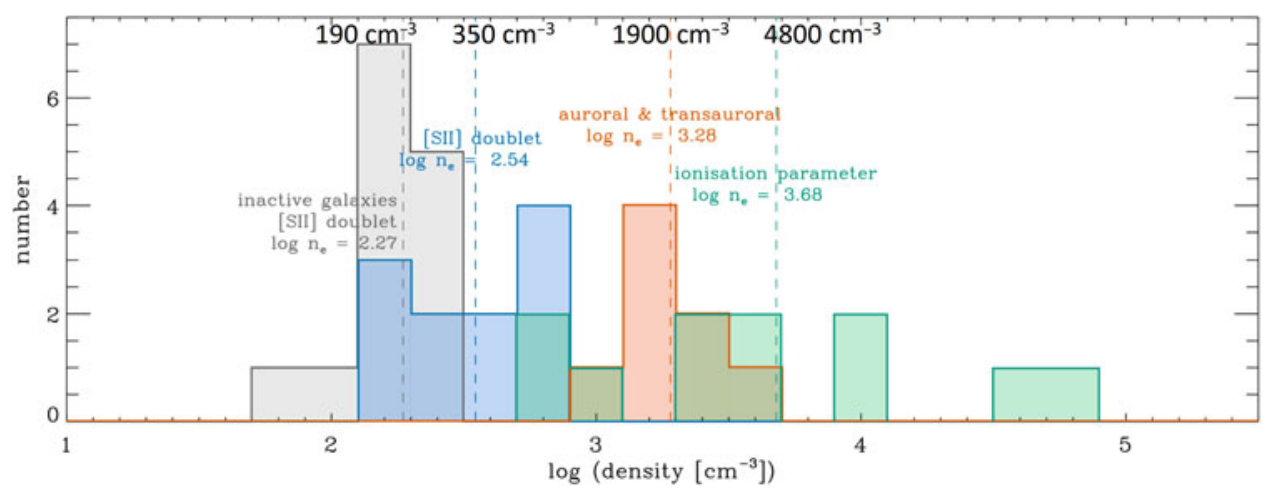

Figure 2. Derived ionized gas densities. Grey: inactive galaxies using the [SII] doublet ratio. Blue: AGN using the doublet ratio. Red: AGN using the auroral/transauroral ratios. Green: AGn using the ionization parameter method.

the AGN luminosity, and of projection effects on the apparent distance from the AGN to the gas. The use of aperture measurements, as is done in this work, will also add to the uncertainty. Our data yield a median density of $n_{\mathrm{e}} \sim 4800 \mathrm{~cm}^{-3}$.

The outcome of this comparison is apparent in Fig. 2, which shows that for these AGN, the [SII] doublet underestimates the true ionized gas density by a factor $5-10$. The reason for this stems from the high energy photons produced by an AGN, which can penetrate deep into clouds, creating a partially ionized zone. As explained by Davies et al. (2020), the ionic fraction of $\mathrm{S}^{+}$remains high even at depths where the fraction of $\mathrm{H}^{+}$is already greatly reduced. As an illustration, in a cloud with $N_{\mathrm{H}}=10^{3} \mathrm{~cm}^{-3}$, only about $25 \%$ of the [SII] emission arises from the fully ionized gas; about half comes from the narrow region around the ionization front where the electron density descreases by a factor 10; and the rest arises from mostly neutral gas deeper in the cloud, where the electron density is only 1-10\% that of the fully ionized gas. Photoionization models suggest that for such a cloud, $n_{\mathrm{e}}$ in the fully ionized gas can be underestimated by as much as a factor 3 when using the [SII] doublet. In denser clouds, the divergence increases rapidly, and can become very extreme. This is reasonably consistent with our empirical finding that for a true density of $2000 \mathrm{~cm}^{-3}$ or more, using the [SII] doublet leads to an underestimate of at least a factor 5 .

As such, one should be very cautious when using the [SII] doublet to estimate the density of gas that is photoionized by an AGN. If the data have high enough signal-tonoise one can use the auroral/transauroral method to obtain a more reliable estimate. Otherwise one should assess the density also using the ionization parameter method.

\section{Implication for outflow rate}

The outflow rate $\dot{M}_{\text {out }}$ depends on the ionized gas mass $M_{\text {out }}$, defined previously, together with the outflow speed $v_{\text {out }}$ and size $r_{\text {out }}$. It is given as $\dot{M}_{\text {out }} \propto M_{\text {out }} v_{\text {out }} / r_{\text {out }}$, where the constant of proportionality is typically taken to be 1 or 3 as described in Lutz et al. (2020). We derive the outflow rate using the [OIII] line luminosity and outflow velocity, setting the outflow size to be the radius of the aperture with a modest correction for projection (since these are Seyfert 2s and hence oriented more edge-on), and adopting the density from the ionization parameter method. The resulting rates are 0.001-0.5 $\mathrm{M}_{\odot} \mathrm{yr}^{-1}$. However, comparing these to published relations such as that of Fiore et al. (2017) is problematic. The reason is that, when compiling their data from the literature, in order to deal with the range of values adopted for $n_{e}$, these authors re-scaled 

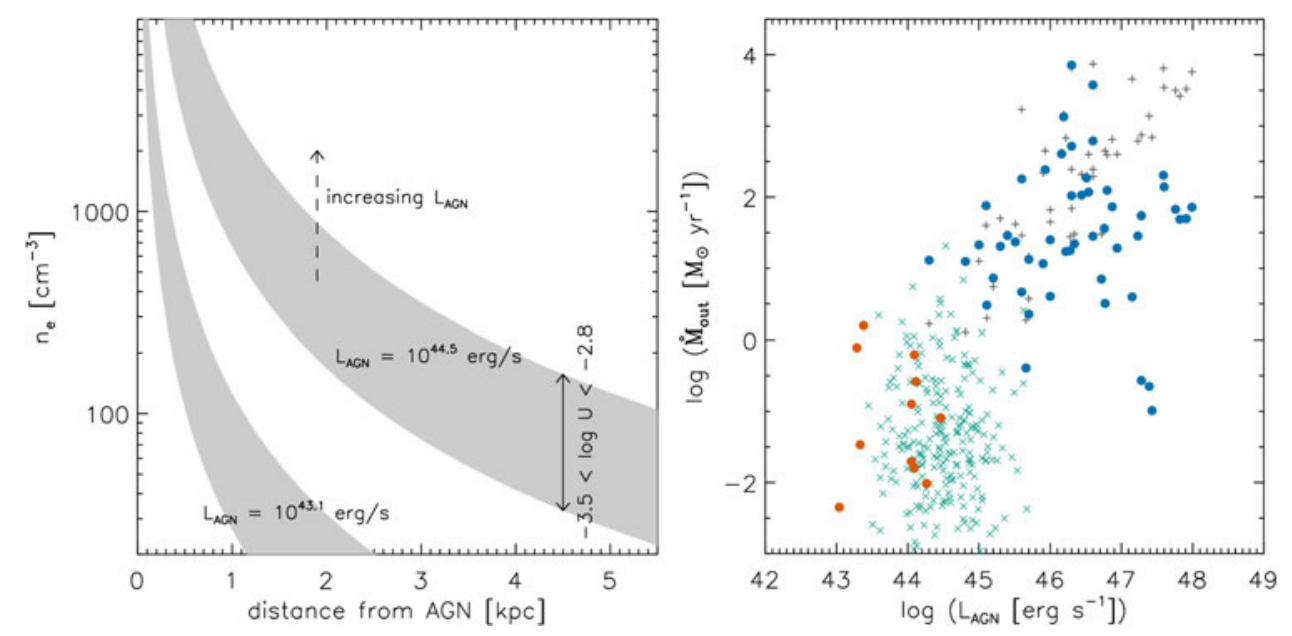

Figure 3. left: Illustration of how inverting the definition of the ionization parameter to give $n_{\mathrm{H}} \propto L_{\mathrm{AGN}} r^{-2} U^{-1}$ means that the gas density at any location in an outflow can be calculated from the AGN luminosity, the distance to the AGN, and the ionization parameter. Examples for 2 luminosities are shown, with the grey region indicative of the uncertainty if the ionization parameter is not known. Right: A modification to the well known $L_{\mathrm{AGN}}-\dot{M}_{\text {out }}$ relation. Black plusses denote the original relation from Fiore et al. (2017). Filled blue circles are our modficiation based on our best estimate of the density. Green crosses are from Baron \& Netzer (2019); and the filled red circles are for the AGN in this study and Davies et al. (2020).

all the rates to a constant value of $\mathrm{n}_{\mathrm{e}}=200 \mathrm{~cm}^{-3}$. This is an order of magnitude different from our typical value.

We therefore explore an alternative representation of the relation: instead of fixing a constant density for all objects, we adopt an estimate of the density based on the ionization parameter method. We use the AGN luminosites $L_{\mathrm{AGN}}$ and outflow sizes $r_{\text {out }}$ given in Fiore et al. (2017). And we adopt a value of $\log U=-2.7$, which is the median for outflows in which both [OIII] and $\mathrm{H} \beta$ luminosities are available. We then make an order-of-magnitude estimate of $n_{\mathrm{e}}$ as illustrated in the left panel of Fig. 3. The key point of this figure is that it emphasizes the enormous variation that can be expected for $n_{\mathrm{e}}$ in different outflows. It highlights how, in the central few hundred parsecs one can find densities in the range $10^{3}-10^{4} \mathrm{~cm}^{-3}$; and that while in some AGN this may fall to $\sim 100 \mathrm{~cm}^{-3}$ at kiloparsec scales, for luminous quasars it may still be in the $\sim 1000 \mathrm{~cm}^{-3}$ range.

The impact on the relation between $\dot{M}_{\text {out }}$ and $L_{\mathrm{AGN}}$ is shown in the right panel of Fig. 3. The black plusses represent data for the original relation published by Fiore et al. (2017). The filled blue circles denote our adjustment to those points, in which we have used our best estimate of the density. The green crosses are from Baron \& Netzer (2019); and the filled red circles are the data for our local luminous AGN in Davies et al. (2020). It can be seen that the scatter of this proposed revision to the relation is rather larger, and the outflow rates are lower by about a factor three.

\section{Conclusions}

We have analysed high signal-to-noise spectra from Xshooter at a resolution of $R \sim$ 10000 covering the central $\sim 300 \mathrm{pc}\left(1.8^{\prime \prime}\right)$ of 11 local luminous AGN $\left(43.0 \leqslant L_{\mathrm{AGN}} \leqslant\right.$ 44.5). And we have measured the density of the ionized gas in three independent ways. In this data the [SII] doublet underestimates the density by a factor $5-10$. It has been known for a long time that in AGN photoionized gas, the low excitation lines ([SII], 
[NII], [OI]) are enhanced in a partially ionized zone where the electron density drops rapidly to $<0.1 n_{\mathrm{H}}$ and the gas becomes mostly neutral. Photoionization models confirm that because of this, the [SII] doublet is unreliable as a tracer of $n_{\mathrm{e}}$ in the fully ionized gas, and so one should be very cautious of using it to estimate density. Instead, if the signal-to-noise is sufficiently high, one can consider the method that uses auroral and transauroral lines. Alternatively, the ionization parameter method can yield an estimate based on $n_{\mathrm{H}} \propto L_{\mathrm{AGN}} /\left(r^{2} U\right)$. The higher densities, $n_{\mathrm{e}} \sim 3000 \mathrm{~cm}^{-3}$, we have found indicate the outflowing ionized gas mass is lower than often reported; but it is also associated with a significant mass of neutral gas. Updating the well known $L_{\mathrm{AGN}}-\dot{M}_{\text {out }}$ relation based on our best estimate of the density, suggests that the scatter is larger than previously thought, and the outflow rates lower.

\section{References}

Baron, D. \& Netzer, H. 2019, MNRAS, 486, 4290

Davies, R., Burtscher, L., Rosario, D., et al. 2015, ApJ, 806, 127

Davies, R., Baron, D., Shimizu, T., et al. 2020, MNRAS, 498, 4150

Dopita, M., Groves, B., Sutherland, R., et al. 2015, ApJ, 806, 127

Fiore, F., Feruglio, C., Shankar, F., et al. 2017, A\&A, 601, A143

Harrison, C., Costa, T., Tadhunter, C., et al. 2018, NatAs, 2, 198

Lutz, D., Sturm, E., Janssen, A., et al. 2020, A\&3A, 633, A134

Holt, J., Tadhunter, C., Morganti, R., et al. 2011, MNRAS, 410, 1527

Somerville, R. \& Davé, R. 2015, ARA\&A, 53, 51

Springel, V., Di Matteo, T., \& Hernquist, L. 2005, MNRAS, 361, 776

Veilleux, S., Maiolino, R., Bolatto, A., et al. 2020, A\&ARv, 28, 2

Vernet, J., Dekker, H., D’Odorico, S., et al. 2011, A\&SA, 536A, 105 